\title{
Wear modelling in railway vehicles: development and implementation of an innovative model for the prediction of the wheel profile evolution due to wear
}

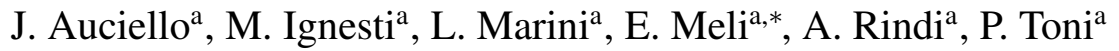 \\ ${ }^{a}$ Department of Energy Engineering, University of Florence, Via S. Marta n. 3, 50139 Firenze, Italy
}

\begin{abstract}
In railway applications, the estimation of the wear in the wheel-rail contact is an important field of study, mainly correlated to the planning of maintenance interventions, vehicle stability and the possibility to carry out specific strategies for the wheel profile optimization.

In this work Authors present a model conceived for the evaluation of the wheel profile evolution due to wear, which is organized in two parts, mutually interactive: a vehicle model for the dynamic analysis and a model for the wear estimation. The first one is a 3D multibody model of a railway vehicle implemented in SIMPACK, a commercial software for the analysis of mechanical systems; the wheel-rail interaction, based on an innovative contact points research method developed by Authors in previous works, is implemented in a C/C++ user routine of SIMPACK. Normal and tangential forces in the contact patches are calculated according to Hertz and Kalker's global theory respectively.

The wear model, developed in MATLAB environment, is mainly based on experimental relationships found in literature between the removed material and the energy dissipated by friction at the contact. It starts from the outputs of the multibody simulations (position of contact points, contact forces and creepages) and estimates the contact pressures by means of a local contact model (FASTSIM algorithm); finally the removed material is calculated and the worn wheel profile is obtained.

This approach allows, evaluating both the quantity of removed material and its distribution along the wheel profile, to analyze the development of the wheel shape during its lifetime. In order to reproduce the wear progression, the overall mileage travelled by the vehicle is divided into discrete steps during which the wheel profile is constant; after simulating one step, the wheel profile is updated by means of the wear model. Therefore, the two models work alternately until completing the whole
\end{abstract}

\footnotetext{
${ }^{*}$ Corresponding author

Email addresses: auciello@mappl.de.unifi.it (J. Auciello), ignesti@mapp1.de.unifi.it (M. Ignesti), marini@mapp1.de.unifi.it (L. Marini), meli@mapp1.de.unifi.it (E. Meli), rindi@mappl.de.unifi.it (A. Rindi), toni@mappl.de.unifi.it (P. Toni)
} 
mileage. The choice of an appropriate length of the step is fundamental in terms of precision and computational time required.

The entire model has been validated in collaboration with Trenitalia S.P.A, which has provided the technical documentation and the experimental results relating to some tests performed with the vehicle DMU ALn 501 "Minuetto" on the Aosta-Pre Saint Didier line.

Keywords: multibody modelling of railway vehicles, wheel-rail contact, wear models

\section{Introduction}

The wear at the wheel-rail interface is an important problem in the railway field. The evolution of the profile shape due to wear has a deep effect on the vehicle dynamics and on its running stability, leading to performance variations both in negotiating curves and in straight track. Therefore the original profiles have to be periodically reestablished by means of turning: particularly, from a safety viewpoint, the arising of a contact geometry which may compromise the vehicle stability or increase the derailment risk has to be avoided. As a matter of fact, vehicle instability could appear even at low speeds in case of high equivalent conicity in the wheel-rail coupling, whereas the derailment may be facilitated by low flange contact angles. A reliable wear model can also be used to optimize the original wheel profiles in order to obtain a more uniform wear. In this way the overall amount of removed material can be reduced so as to increase the mean time between two maintenance intervals and, at the same time, the dynamical performance of the wheel-rail pair can be kept approximately constant in the time.

It is important to underline that one of the most critical aspect in the development of a wear model is the availability of experimental results, since the collection of the data requires some months with relevant economic cost. If on line experimental measurement cannot be carried out, the problem could be overcome using tools provided by softwares [1] or carrying out experimental proofs on a scaled test rig [2].

In this work the authors will present a procedure to estimate the evolution of the wheel profile due to wear based on a model that combines multibody and wear modeling. More specifically, the general layout of the model consists of two parts mutually interactive: the vehicle model (multibody model and 3D global contact model) and the wear model (local contact model, wear evaluation and profiles update). The multibody model, implemented in the SIMPACK environment, accurately reproduces the dynamics of the vehicle, taking into account all the significant degrees of freedom. The 3D global contact model, implemented in $\mathrm{C} / \mathrm{C}++$ environment and developed by the authors in previous works [3], detects the wheel-rail contact points by means of an innovative algorithm based on a fully 3D semi-analytic procedures and then, for each contact point, calculates the contact forces through Hertz's and Kalker's theory [4]. 


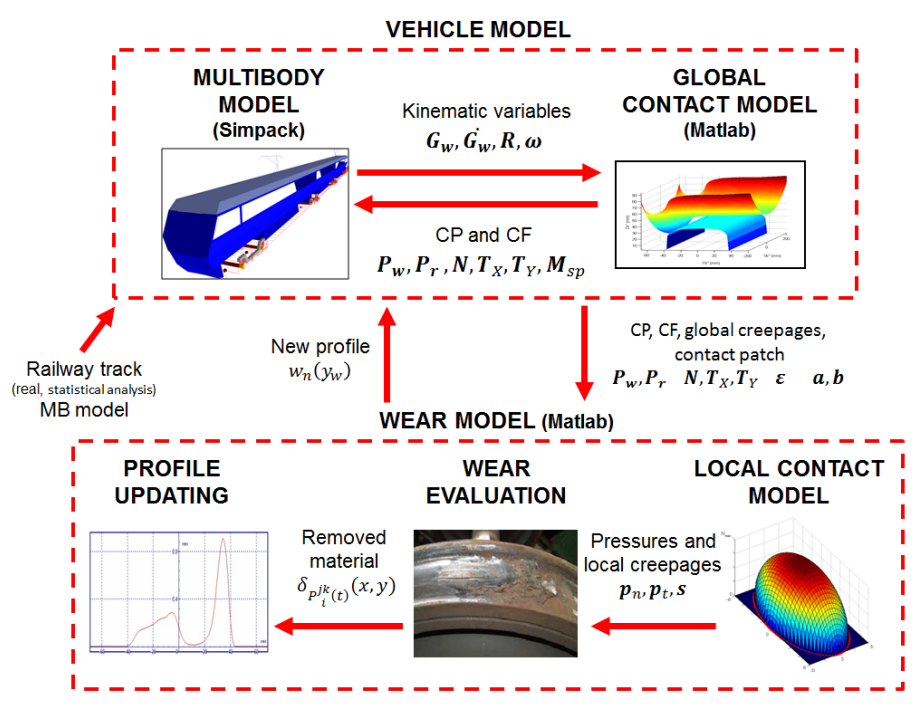

Figure 1: General Architecture of the Model

As regards the wear estimation, the local contact model uses the outputs of the multibody simulations (contact points, contact forces and global creepages), to calculate the contact pressures and the local creapages inside the contact patch, while the wear model, thanks to these quantities, evaluates the total amount of removed material due to wear and its distribution along the wheel profile. The whole procedure for the wear estimation is implemented in MATLAB environment.

In this work the entire model has been validated by means of the experimental data provided by Trenitalia S.p.A. and RFI; the data concern the Aosta-Pre Saint Didier railway line and the vehicle ALSTOM DMU Aln 501 Minuetto which, in this scenery, exhibits serious problems in terms of wear.

\section{Layout of the Model}

The general architecture of the whole model is shown in the block diagram in Fig. 1: it includes two main parts that work alternatively during each step.

The vehicle model represents the part which is responsible for the dynamic simulation and it is made up of the multibody model and of the global contact model; the two subsystems interact online to each other during the simulations creating a loop, as to reproduce the vehicle dynamics. The wear model, instead, is made up of three subparts: the local contact model, the wear evaluation and the profile updating procedure.

In more detail, the multibody model implemented exchanges data continuously at each time simulation step with the global contact model [3][5], passing the wheelset kinematic variables (wheelset position and orientation and their derivatives) and receiving the positions of the contact points, the wheel-rail contact forces and the global creepages.

The main inputs of the vehicle model are the railway track and the multibody model 
of the considered vehicle: in this research activity, according to the specifications required by Trenitalia and considering the complexity and the length of the considered railway track, a statistical approach is necessary to achieve general significant results in a reasonable time. For these reasons the entire Aosta-Pre Sain Didier line has been substituted with an equivalent set of different curved tracks, classified by radius, superelevation and traveling speed, which has been built consulting a detailed track database provided by Rete Ferroviaria Italiana.

Once the multibody simulations are completed, the local contact model, (based on the FASTSIM algorithm [4]) evaluates, starting from the global contact variables, the contact pressures, the local creepages inside each detected contact patch and consequently divedes it into adhesion area and creep area. Then, the distribution of removed material (hypothesizing the contact in dry conditions as required by Trenitalia and RFI) is calculated on the wheel surface only within the creep area using a experimental law between the removed material and the energy dissipated by friction at the contact interface [2][6]. Finally, the wheel profile is updated through suitable numerical procedures and rapresent the output of one discrete step of the whole model loop.

The evolution of the wheel profile is therefore treated with a discrete approach. The entire mileage to be simulated is divided in a few spatial steps, within the profile is maintained constant during the dynamic simulations; the results of the wear model, at the end of the current step, allow to update the wheel profile for the next step of the procedure. The step length depends on the total distance to be covered and it is one of most important aspect of the entire numerical procedure, because it directly affects the precision: in fact, the smaller the step is, the higher the accuracy and the overall computation time are; hence the choice has to be a compromise between these aspects. Moreover, from a numerical point of view, the step length can be chosen either constant during the overall distance or variable (introducing for example a threshold on the maximum of removed material); nevertheless, since the wear progress is almost linear with respect to the traveled distance, the constant step length turns out to be a quite suitable choice for this kind of problem, providing comparable results in terms of accuracy and better performance in terms of numerical efficiency.

\section{The Vehicle Model}

In this section a brief description of the vehicle model, made up of the multibody model and the global contact model, is given.

\subsection{The Multibody Model}

The DMU Aln 501 Minuetto has been chosen as benchmark vehicle for this research; the physical and geometrical characteristics of the vehicle can be found in literature [7]. It is made up of three coaches and four bogies with two wheelsets; the external bogies are motorized whereas the two intermediate trailer bogies are of Jacobs type, shared between two coaches. The multibody model has been realized in the 
SIMPACK environment (see Fig. 2) and consists of thirty-one rigid bodies: 3 coaches, 4 bogies, 8 wheelsets and 16 axleboxes. The most significant inertial properties of the model bodies are summarized in Tab. 1 .

\begin{tabular}{|c|c|c|c|c|}
\hline MBS body & $\begin{array}{c}\text { Mass } \\
(\mathrm{kg})\end{array}$ & $\begin{array}{c}\text { Roll Inertia } \\
\left(\mathrm{kg} \cdot \mathrm{m}^{2}\right)\end{array}$ & $\begin{array}{c}\text { Pitch Inertia } \\
\left(\mathrm{kg} \cdot \mathrm{m}^{2}\right)\end{array}$ & $\begin{array}{c}\text { Yaw Inertia } \\
\left(\mathrm{kg} \cdot \mathrm{m}^{2}\right)\end{array}$ \\
\hline \hline External coach & 31568 & 66700 & 764000 & 743000 \\
Internal coach & 14496 & 30600 & 245000 & 236000 \\
Motor bogie frame & 3306 & 1578 & 2772 & 4200 \\
Trailer bogie frame & 3122 & 1674 & 3453 & 5011 \\
\hline
\end{tabular}

Table 1: Inertia properties of the multibody model

The rigid bodies are connected by means of appropriate elastic and damping elements; particularly the vehicle, as in the most part of passenger trains, is equipped with two suspension stages. The primary suspensions link the axlebox to the bogies (see Fig. 3) and comprise two springs and two vertical dampers, while the secondary suspensions connect the bogies to the coaches (see Fig. 4) and comprise the following elements:

- two air springs;

- six dampers (lateral, vertical and anti - yaw dampers);

- one traction rod;

- the roll bar (not visible in the figure);

- two lateral bumpstops.

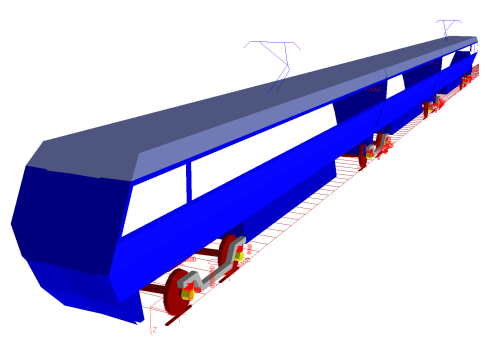

Figure 2: Multibody model

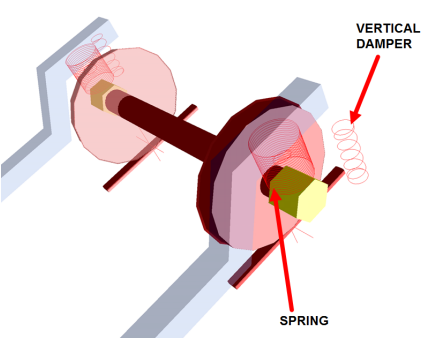

Figure 3: Primary suspensions

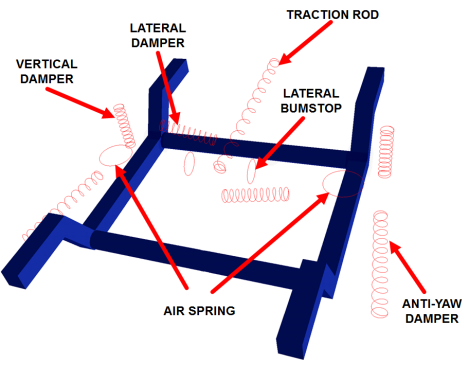

Figure 4: Secondary suspensions

Both the stages of suspensions have been modelled by means of three-dimensional viscoelastic force elements taking into account all the mechanical non linearities (bumpstop clearance, dampers and rod behavior). The main linear characteristics of the suspensions are shown in Tab. 2.

\subsection{The Global Contact Model}

Dynamic simulations of railway vehicles need a reliable and efficient method to evaluate the contact points at wheel-rail interface, because their position has a considerable influence both on the direction and on the magnitude of the contact forces. In this work a specific contact model has been considered instead of that implemented in SIMPACK in order to achieve better reliability and accuracy. The proposed contact 


\begin{tabular}{|c|c|c|c|c|c|c|}
\hline $\begin{array}{c}\text { MBS } \\
\text { Element }\end{array}$ & $\begin{array}{c}\text { Longitudinal } \\
\text { Stiffness } \\
(\mathrm{N} / \mathrm{m})\end{array}$ & $\begin{array}{c}\text { Lateral } \\
\text { Stiffness } \\
(\mathrm{N} / \mathrm{m})\end{array}$ & $\begin{array}{c}\text { Vertical } \\
\text { Stiffness } \\
(\mathrm{N} / \mathrm{m})\end{array}$ & $\begin{array}{c}\text { Roll } \\
\text { Stiffness } \\
(\mathrm{Nm} / \mathrm{rad})\end{array}$ & $\begin{array}{c}\text { Pitch } \\
\text { Stiffness } \\
(\mathrm{Nm} / \mathrm{rad})\end{array}$ & $\begin{array}{c}\text { Yaw } \\
\text { Stiffness } \\
(\mathrm{Nm} / \mathrm{rad})\end{array}$ \\
\hline \hline $\begin{array}{c}\text { Primary } \\
\text { Suspension } \\
\text { Spring }\end{array}$ & 1259600 & 1259600 & 901100 & 10800 & 10800 & 1000 \\
\hline $\begin{array}{c}\text { Secondary } \\
\text { Suspension } \\
\text { Air Spring }\end{array}$ & 120000 & 120000 & 398000 & - & - & - \\
\hline $\begin{array}{c}\text { Secondary } \\
\text { Suspension } \\
\text { Roll Bar }\end{array}$ & - & - & - & 2600000 & - & - \\
\hline
\end{tabular}

Table 2: Main linear stiffness properties of the suspensions

model is divided in two parts: in the first one the contact points are detected by means of an innovative algorithm developed by the authors in previous works [3] [5], while in the second one the global contact forces acting at the wheel-rail interface are evaluated by means of Hertz's and Kalker's global theories [4]. The new model is based on a semianalytic approach that guarantees the following features:

- generic wheel and rail profiles can be implemented;

- fully 3D handling of the contact problem, with all degrees of freedom between wheel and rail taken into account;

- no simplifying hypotheses on the problem geometry and kinematics;

- multiple points of contact are allowed with no bounds to the their overall number;

- high numerical efficiency which allows the online implementation directly within the multibody models, without look-up tables; numerical performance better than those obtainable with commercial softwares (Vi-Rail ${ }^{\mathrm{TM}}$, SIMPACK) [3][5].

Two specific reference systems have to be introduced in order to simplify the wheel and rail profiles description and consequently the model's equations: the auxiliary reference system and the local reference system. The auxiliary system $O_{r} x_{r} y_{r} z_{r}$ moves along the track centerline following the wheelset during the dynamic simulations: the $x_{r}$ axis is tangent to the center line of the track in the origin $O_{r}$, whose position is defined so that the $y_{r} z_{r}$ plane contains the center of mass $\mathbf{G}_{\mathbf{w}}$ of the wheelset, and the $z_{r}$ axis is perpendicular to plane of track. The local system $O_{w} x_{w} y_{w} z_{w}$ is fixed on the wheelset except for the rotation around its axis and the $x_{w}$ axis is parallel to the $x_{r} y_{r}$ plane (see Fig. 5(b)). In the following, for the sake of simplicity, the variables referred to the local system will be marked with the apex $w$, while those referred to the auxiliary system with the apex $r$; the variables belonging to the wheel and to the rail will be indicated with the subscripts $w$ and $r$ respectively.

\subsubsection{The Distance Method Algorithm}

In this subsection the algorithm used for detecting the contact points will be described. The main innovative aspect of the algorithm is the reduction of the original multi-dimensional contact problem (4D) to a simple scalar problem (1D), which can be easily handled by means of numerical methods, with remarkable advantages:

- the multiple solution management is simpler;

- a wide range of algorithms, even the elementary non-iterative ones, can efficiently resolve the numerical problem; 


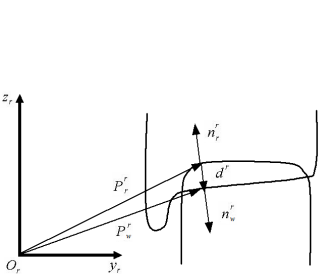

(a)

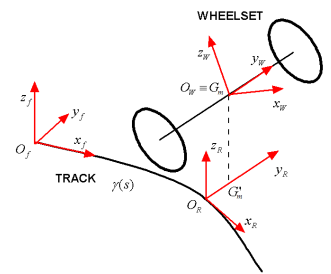

(b)

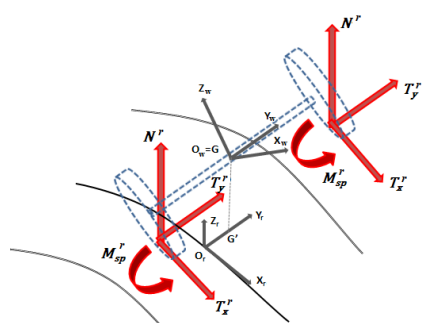

Figure 6: Global contact forces

Figure 5: Distance method

- the convergence can be easily achieved and the algorithm converges to the solutions with fewer iterations and less computational effort.

The distance method algorithm (see Fig. 5(a)) starts from a classical formulation of the contact problem in multibody field; considering the reference systems adopted, the following geometrical conditions hold:

- the normal unitary vector relative to the rail surface $\mathbf{n}_{r}^{r}\left(\mathbf{P}_{r}^{r}\right)$ and the wheel surface unitary vector $\mathbf{n}_{w}^{r}\left(\mathbf{P}_{w}^{r}\right)$ have to be parallel ( $\mathbf{R}_{2}$ is the rotation matrix that links the local system to the auxiliary one):

$$
\mathbf{n}_{r}^{r} \times \mathbf{n}_{w}^{r}\left(\mathbf{P}_{w}^{r}\right)=\mathbf{n}_{r}^{r}\left(\mathbf{P}_{r}^{r}\right) \times \mathbf{R}_{2} \mathbf{n}_{w}^{w}\left(\mathbf{P}_{w}^{w}\right)=\mathbf{0}
$$

the wheel and rail surfaces can be locally considered as revolution and extrusion surface respectively: $\mathbf{P}_{w}^{w T}=\left(x_{w}, y_{w},-\sqrt{w\left(y_{w}\right)^{2}-x_{w}^{2}}\right), \mathbf{P}_{r}^{r T}=\left(x_{r}, y_{r}, r\left(y_{r}\right)\right)$, where the generative function $w\left(y_{w}\right)$ and $r\left(y_{r}\right)$ are supposed to be known;

- the rail surface normal unitary vector $\mathbf{n}_{r}^{r}\left(\mathbf{P}_{r}^{r}\right)$ has to be parallel to the distance vector $\mathbf{d}^{r}=\mathbf{P}_{w}^{r}\left(x_{w}, y_{w}\right)-\mathbf{P}_{r}^{r}\left(x_{r}, y_{r}\right)$ between the generic point of the wheel and of the rail:

$$
\mathbf{n}_{r}^{r}\left(\mathbf{P}_{r}^{r}\right) \times \mathbf{d}^{r}=\mathbf{0} .
$$

Alternately, the problem can also be equivalently formulated imposing that the distance vector $\mathbf{d}^{r}$ is perpendicular both to the wheel and to the rail tangent plane (respectively $\pi_{w}\left(P_{r}^{r}\right)$ and $\left.\pi_{r}\left(P_{w}^{r}\right)\right)$. Nevertheless, due to the particular structure of the algebraic equations, the calculation and the resolution algorithm are more complicated than the ones arising from equations (1) and (2).

The distance between the generic points on the wheel and on the rail can be expressed as

$$
\mathbf{d}^{r}\left(x_{w}, y_{w}, x_{r}, y_{r}\right)=\mathbf{O}_{w}^{r}+R_{2} \mathbf{P}_{w}^{w}\left(x_{w}, y_{w}\right)+\mathbf{P}_{r}^{r}\left(x_{r}, y_{r}\right)
$$

thus, it depends on the four parameters $\left(x_{w}, y_{w}, x_{r}, y_{r}\right)$, that identify a point on both the surfaces. The equations (1) and (2) constitute a system with six scalar equations and four unknowns $\left(x_{w}, y_{w}, x_{r}, y_{r}\right)$ (only four of the equations are independent). As stated previously, the 4D problem can be reduced to a scalar equation in the unknown $y_{w}$ expressing $x_{w}, x_{r}$, and $y_{r}$ as functions of $y_{w}$. The second component of equation (1) leads to the following equation:

$$
r_{13} \sqrt{w\left(y_{w}\right)^{2}-x_{w}^{2}}=r_{11} x_{w}-r_{12} w\left(y_{w}\right) w^{\prime}\left(y_{w}\right)
$$


where $r_{i j}$ are the elements of the $R_{w}^{r}$ matrix. Calling $A=r_{13}, B=w\left(y_{w}\right), C=r_{11}$ and $D=r_{12} w\left(y_{w}\right) w^{\prime}\left(y_{w}\right)$, removing the radical and solving for $x_{w}$ the previous equation becomes:

$$
x_{w 1,2}\left(y_{w}\right)=\frac{C D \pm \sqrt{C^{2} D^{2}-\left(A^{2}+C^{2}\right)\left(D^{2}-A^{2} B^{2}\right)}}{A^{2}+C^{2}}
$$

as can be seen, there are two possible values of $x_{w}$ for each $y_{w}$.

From the first component of the equation (1) the following relation for $r^{\prime}\left(y_{r}\right)$ holds:

$$
r^{\prime}\left(y_{r}\right)_{1,2}=\frac{r_{21} x_{r 1,2}\left(y_{w}\right)-r_{22} w\left(y_{w}\right) w^{\prime}\left(y_{w}\right)-r_{23} \sqrt{w\left(y_{w}\right)^{2}-x_{w 1,2}\left(y_{w}\right)^{2}}}{r_{32} w\left(y_{w}\right) w^{\prime}\left(y_{w}\right)+r_{33} \sqrt{w\left(y_{w}\right)^{2}-x_{w 1,2}\left(y_{w}\right)^{2}}} .
$$

By the second component of equation (2) the expression of $x_{r 1,2}\left(y_{w}\right)$ can be obtained:

$$
x_{r 1,2}\left(y_{w}\right)=r_{11} x_{w 1,2}\left(y_{w}\right)+r_{12} y_{w}-r_{13} \sqrt{w\left(y_{w}\right)^{2}-x_{w 1,2}\left(y_{w}\right)^{2}} .
$$

Finally, replacing the variables $x_{w 1,2}\left(y_{w}\right), x_{r 1,2}\left(y_{w}\right)$ and $y_{r 1,2}\left(y_{w}\right)$ in the first component of equation (2), the following 1D scalar equation can be written:

$$
\begin{gathered}
F_{1,2}\left(y_{w}\right)=-r^{\prime}\left[G_{m z}+r_{32} y_{w}-r_{33} \sqrt{w^{2}-x_{w 1,2}^{2}}-b\right]+ \\
-\left[G_{m y}+r_{21} x_{w 1,2}+r_{22} y_{w}-r_{23} \sqrt{w^{2}-x_{w 1,2}^{2}}-y_{r 1,2}\right]=0
\end{gathered}
$$

where $G_{w x}, G_{w y}, G_{w z}$ are the coordinates of the wheelset center of mass in the auxiliary system. The expression (8) consists of two scalar equations in the variable $y_{w}$ easy to resolve numerically with the advantages previously mentioned. Thus, once obtained the generic solution (indicated with the subscript $i$ ) $y_{w i}$ of equation (8), the values of the unknowns $\left(x_{w i}, y_{w i}, x_{r i}, y_{r i}\right)$ and consequently the contact points $\mathbf{P}_{w i}^{r}=\mathbf{P}_{w}^{r}\left(x_{w i}, y_{w i}\right)$ and $\mathbf{P}_{r i}^{r}=\mathbf{P}_{r}^{r}\left(x_{r i}, y_{r i}\right)$ can be found by substitution.

Since the equations (1) (2) contain irrational terms, the generic solution $\left(x_{w i}, y_{w i}\right.$, $\left.x_{r i}, y_{r i}\right)$ must satisfy the following analytical conditions:

- the solution must be real;

- the solution does not have to generate complex terms (that could be caused by the radicals in the equations);

- $\left(x_{w 1 i}, y_{w 1 i}\right)$ and $\left(x_{w 2 i}, y_{w 2 i}\right)$ must be effective solutions of the equation (4) (check necessary because of the radical removal by squaring).

The following physical conditions have also to be respected so that the contact is physically possible:

- the penetration between the wheel and rail surfaces $\left(\tilde{p}_{n}=\mathbf{d}^{r} \cdot \mathbf{n}_{r}^{r}\right)$ have to be less or equal to zero, according to the adopted nomenclature;

- multiple solutions have to be rejected;

- the normal curvatures of the wheel and rail surfaces in the longitudinal and lateral direction $\left(k_{1, w i}, k_{1, r i}, k_{2, r i}, k_{2, r i}\right)$, evaluated in the contact points, have to satisfy the convexity condition in order to make the contact physically possible $\left(k_{1, w i}+k_{1, r i}>\right.$ $\left.0 ; k_{2, w i}+k_{2, r i}>0\right)$. 


\subsubsection{The Contact Forces}

The calculation of the contact forces for each contact point is based on a semielastic approach which uses both Hertz and Kalker's global theories (see Fig. 6). The normal contact forces, according to the Hertz theory, depend both on the penetration between $\left(\tilde{p}_{n}\right)$ the surface of the wheel and rail and the penetration velocity $v_{n}$ [4]:

$$
N^{r}=\left[-k_{h}\left|\tilde{p}_{n}\right|^{\gamma}+k_{v}\left|v_{n}\right| \frac{\operatorname{sign}\left(v_{n}\right)-1}{2}\right] \frac{\operatorname{sign}\left(\tilde{p}_{n}\right)-1}{2}
$$

where $\gamma$ is equal to $3 / 2, k_{h}$ is a Kalker's stiffness costant, $k_{v}$ is a damping contact constant [4].

The global creepages $\varepsilon$ (longitudinal $\varepsilon_{x}$, lateral $\varepsilon_{y}$ and spin creepage $\varepsilon_{s p}$ ) are calculated as follows:

$$
\varepsilon_{x}=\frac{\mathbf{V} \bullet \mathbf{i}_{r}}{\left|\dot{\mathbf{G}}_{w}^{r}\right|} \quad, \quad \varepsilon_{y}=\frac{\mathbf{V} \bullet \mathbf{t}_{r}^{r}\left(\mathbf{P}_{r}^{r}\right)}{\left|\dot{\mathbf{G}}_{w}^{r}\right|} \quad, \quad \varepsilon_{s p}=\frac{\boldsymbol{\omega}_{w}^{r} \bullet \mathbf{n}_{r}^{r}\left(\mathbf{P}_{r}^{r}\right)}{\left|\dot{\mathbf{G}}_{w}^{r}\right|}
$$

where $\mathbf{V}$ is the velocity of contact point rigidly connected to the wheelset, $\dot{\mathbf{G}}_{w}^{r}$ is the wheelset center of mass velocity (taken as the reference velocity for the calculation of the global creepages), $\boldsymbol{\omega}_{w}^{r}$ is the angular velocity of wheelset expressed in auxiliary system, $\mathbf{i}_{r}$ is the unit vector in longitudinal direction of the auxiliary system and $\mathbf{t}_{r}^{r}$ is the tangential unit vector to the rail profile.

The tangential contact forces $\tilde{T}_{x}^{r}, \tilde{T}_{y}^{r}$ and the spin torque $M_{s p}^{r}$ (expressed in the auxiliary system) are calculated by means of the Kalker's global theory:

$$
\tilde{T}_{x}^{r}=-f_{11} \varepsilon_{x} \quad, \quad \tilde{T}_{y}^{r}=-f_{22} \varepsilon_{y}-f_{23} \varepsilon_{s p} \quad, \quad M_{s p}^{r}=f_{23} \varepsilon_{y}-f_{33} \varepsilon_{s p}
$$

where the coefficients $f_{i j}$ are function both of the materials and of the semiaxes of the contact patch:

$$
f_{11}=a b G C_{11} \quad, \quad f_{22}=a b G C_{22} \quad, \quad f_{23}=(a b)^{3 / 2} G C_{23} \quad, \quad f_{33}=(a b)^{2} G C_{33}
$$

in which $G$ is the wheel and rail combined shear modulus and $C_{i j}$ are the Kalker's coefficients that can be found tabulated in literature [4].

Since the Kalker's theory is linear, to include the effect of the adhesion limit due to friction, a saturation criterion has to be introduced in the model to limit the magnitude of the tangential contact force $\tilde{T}^{r}=\sqrt{\tilde{T}_{x}^{r^{2}}+\tilde{T}_{y}^{r^{2}}}$ which cannot exceed the slip value $T^{r}=\mu N^{r}$. Therefore, a saturation coefficient $\epsilon_{\text {sat }}$ is defined as follows [8][9]:

$$
\epsilon_{\text {sat }}=\left\{\begin{array}{ll}
\frac{\mu_{c} N^{r}}{\tilde{T}^{r}}\left[\left(\frac{\tilde{T}^{r}}{\mu_{c} N^{r}}\right)-\frac{1}{3}\left(\frac{\tilde{T}^{r}}{\mu_{c} N^{r}}\right)^{2}+\frac{1}{27}\left(\frac{\tilde{T}^{r}}{\mu_{c} N^{r}}\right)^{3}\right] & \text { if } \tilde{T}^{r} \leq 3 \mu_{c} N^{r} \\
\frac{\mu_{c} N^{r}}{\tilde{T}^{r}} & \text { if } \tilde{T}^{r}>3 \mu_{c} N^{r}
\end{array} ;\right.
$$

in this way the saturated tangential force will be $\mathbf{T}^{r}=\epsilon_{\text {sat }} \tilde{\mathbf{T}}^{r}$. 


\section{The Wear Model}

In this section the three phases of the wear model will be described in details: the local contact model, the evaluation of the amount of removed material due to wear and the wheel profile update.

\subsection{The Local Contact Model}

The purpose of the local contact model is the calculation of the local contact variables into the contact patch (normal and tangential contact pressures $p_{n}, \mathbf{p}_{t}$ and local creep s) starting from the corresponding global variables (contact points $\mathbf{P}_{w}^{r}, \mathbf{P}_{r}^{r}$, contact forces $N^{r}, T_{x}^{r}, T_{y}^{r}$, global creepage $\varepsilon$ and semiaxes of the contact patch $a, b$ ).

This model is based on the Kalker's local theory in the simplified version implemented in the algorithm FASTSIM; this algorithm contains an extremely efficient version (although necessarily approximate) of the Kalker theory commonly used in railway field [4].

For the local analysis a new reference system is defined at the wheel-rail interface on the contact plane (i.e. the common tangent plane between the wheel and rail surfaces). The $x$ and $y$ axes are the longitudinal and the transversal direction of the contact plane respectively (see Fig. 7, 9); therefore, they are not parallel to neither the local reference system of the wheelset nor the auxiliary system. The working hypotesis on which the algorithm is developed is the proportionality between the tangential contact pressure $\mathbf{p}_{t}$ and the elastic displacements $\mathbf{u}$, in a generic point of the contact patch:

$$
\mathbf{u}(x, y)=L \mathbf{p}_{t}(x, y), \quad L=L(\varepsilon, a, b, G, \nu)
$$

where the flexibility $L$ is a function of the global creepages $\varepsilon$, the semiaxes of the contact patch $a, b$, the wheel and rail combined shear modulus $G$ and the wheel and rail combined Poisson's ratio $\nu$ [4].

The local creepages $\mathbf{s}$ can be calculated deriving the elastic displacements and considering both the rigid global creepages and the vehicle speed $V=\left\|\dot{\mathbf{G}}_{w}^{r}\right\|$ :

$$
\mathbf{s}(x, y)=\dot{\mathbf{u}}(x, y)+V\left(\begin{array}{l}
\varepsilon_{x} \\
\varepsilon_{y}
\end{array}\right)
$$

At this point it is necessary to discretize the elliptical contact patch in a grid of points in which the quantities $p_{n}, \mathbf{p}_{t}$ and $\mathbf{s}$ will be evaluated. Initially the transversal axis (respect to the motion direction) of the contact ellipse has been divided in $n_{y}-1$ equal parts of magnitude $\Delta y=2 b /\left(n_{y}-1\right)$ by means of $n_{y}$ equidistant nodes. Then the longitudinal sections of the patch (long $2 a(y)=2 a \sqrt{1-y / b^{2}}$ ) have been divided in $n_{x}-1$ equal parts of magnitude $\Delta x(y)=2 a(y) /\left(n_{x}-1\right)$ by means of $n_{x}$ equidistant nodes (see Fig. 7). This choice leads to a longitudinal resolution that is not constant and increases in the nearby of the lateral edges of the ellipse, where the length $a(y)$ are shorter. This procedure provides more accurate results near the edges of the ellipse, where a constant resolution grid would generate excessive numerical noise. The values 
of the $n_{x}$ and $n_{y}$ parameters have to assure the right balance between precision and computational load; good values of compromise are in the range [25 50].

Once the contact patch is discretized, the FASTSIM algorithm allows to iteratively evaluate both the contact pressures value $p_{n}, \mathbf{p}_{t}$ and the local creepage $\mathbf{s}$ in order to divide the contact patch in adhesion and slip zone. Indicating the generic point of the grid with $\left(x_{i}, y_{j}\right), 1 \leq i \leq n_{x} 1 \leq j \leq n_{y}$, the normal contact pressure can be expressed as:

$$
p_{n}\left(x_{i}, y_{j}\right)=\frac{3}{2} \frac{N^{r}}{\pi a b} \sqrt{1-\frac{x_{i}^{2}}{a^{2}}-\frac{y_{j}^{2}}{b^{2}}}
$$

where $N^{r}$ is the normal contact force, while the limit adhesion pressure $\mathbf{p}_{A}$ is:

$$
\mathbf{p}_{A}\left(x_{i}, y_{j}\right)=\mathbf{p}_{t}\left(x_{i-1}, y_{j}\right)-\left(\begin{array}{c}
\varepsilon_{x} \\
\varepsilon_{y}
\end{array}\right) \frac{\Delta x\left(y_{j}\right)}{L}
$$

thus, knowing the variable values in the point $\left(x_{i-1}, y_{j}\right)$, it is possible to pass to the point $\left(x_{i}, y_{j}\right)$ as follows:

$$
\begin{gathered}
\text { if }\left\|\mathbf{p}_{A}\left(x_{i}, y_{j}\right)\right\| \leq \mu p_{n}\left(x_{i}, y_{j}\right) \Rightarrow\left\{\begin{array}{c}
\mathbf{p}_{t}\left(x_{i}, y_{j}\right)=\mathbf{p}_{A}\left(x_{i}, y_{j}\right) \\
\mathbf{s}\left(x_{i}, y_{j}\right)=\mathbf{0}
\end{array}\right. \\
\text { if }\left\|\mathbf{p}_{A}\left(x_{i}, y_{j}\right)\right\|>\mu p_{n}\left(x_{i}, y_{j}\right) \Rightarrow\left\{\begin{array}{c}
\mathbf{p}_{t}\left(x_{i}, y_{j}\right)=\mu p_{n}\left(x_{i}, y_{j}\right) \mathbf{p}_{A}\left(x_{i}, y_{j}\right) /\left\|\mathbf{p}_{A}\left(x_{i}, y_{j}\right)\right\| \\
\mathbf{s}\left(x_{i}, y_{j}\right)=\frac{L V}{\Delta x\left(y_{j}\right)}\left(\mathbf{p}_{t}\left(x_{i}, y_{j}\right)-\mathbf{p}_{A}\left(x_{i}, y_{j}\right)\right)
\end{array}\right.
\end{gathered}
$$

where $\mu$ is the static friction coefficient; equations (18a) and (18b) hold respectively in the adhesion and slip zone.

Iterating the procedure for $2 \leq i \leq n_{x}$ and successively for $1 \leq j \leq n_{y}$ and assuming as boundary conditions $\mathbf{p}_{t}\left(x_{1}, y_{j}\right)=\mathbf{0}, \mathbf{s}\left(x_{1}, y_{j}\right)=\mathbf{0}$ for $1 \leq j \leq n_{y}$ (i.e. pressures and creepages zero out of the contact patch), the desired distribution of $p_{n}\left(x_{i}, y_{j}\right), \mathbf{p}_{t}\left(x_{i}, y_{j}\right)$ and $\mathbf{s}\left(x_{i}, y_{j}\right)$ can be determined.

\subsection{The Wear Evaluation}

The following working hypothesis have been considered to evaluate the distribution of removed material on the wheel, in agreement with Trenitalia and RFI requests:

- the wear affects only the wheels, while the rails keeps their initial profiles that are a family of worn profiles (provided by RFI) each characteristic of a single curve of the statistical analysis;

- the output of the wear model is the mean wheel profile to be used in the next step, which include the effect of the wear on all the wheels of the considered vehicle;

- dry conditions in the wheel-rail interface.

The calculation of the wear is based on a experimental relationship between the volume of removed material and the frictional work [2][6]: particularly the used relationship is able to directly evaluate the specific volumes of removed material $\delta_{P_{w i}^{j k}(t)}(x, y)$ 
related to the $\mathrm{i}$-th contact points $P_{w i}^{j k}(t)$ on the $\mathrm{j}$-th wheel and rail pair during the $\mathrm{k}$-th of the $N_{c}$ dynamic simulations.

The calculation of $\delta_{P_{w i}^{j k}(t)}(x, y)$ requires first of all the evaluation of the friction power developed by the tangential contact stresses; to this aim the wear index $I_{W}$ (expressed in $\mathrm{N} / \mathrm{mm}^{2}$ ) is defined as follows:

$$
I_{W}=\frac{\mathbf{p}_{t} \bullet \mathbf{s}}{V} .
$$

This index is experimentally correlated with the wear rate $K_{W}$ (expressed in $\mu \mathrm{g} /(\mathrm{m}$. $\left.m m^{2}\right)$ ) which represents the mass of removed material for unit of distance traveled by the vehicle (expressed in $m$ ) and for unit of surface (expressed in $\mathrm{mm}^{2}$ ). Wear tests carried out in the case of metal-metal contact with dry surfaces using a twin disc test machine can be found in literature [2]. The experimental relationship between $K_{W}$ and $I_{W}$ adopted for the wear model described in this work is the following (see Fig. 8):

$$
K_{W}\left(I_{W}\right)=\left\{\begin{array}{cc}
5.3 * I_{W} & I_{W}<10.4 \\
55.12 & 10.4 \leq I_{W} \leq 77.2 \\
61.9 * I_{W}-4723.56 & I_{W}>77.2
\end{array}\right.
$$

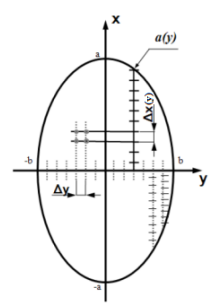

Figure 7: Contact patch discretization

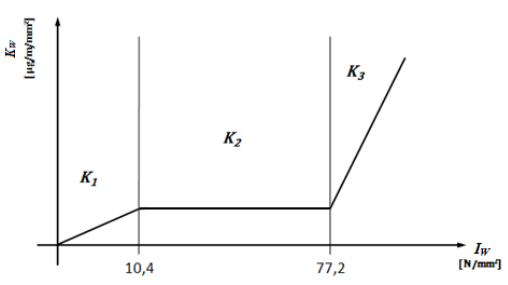

Figure 8: Trend of the wear rate $K_{W}$

After the evaluation of the wear rate $K_{W}\left(I_{W}\right)$, the specific volume of removed material on the wheel (for unit of distance traveled by the vehicle and for unit of surface) can be calculated as follows (expressed in $\mathrm{mm}^{3} /\left(\mathrm{m} \cdot \mathrm{mm}^{2}\right)$ ):

$$
\delta_{P_{w i}^{j k}(t)}(x, y)=K_{W}\left(I_{W}\right) \frac{1}{\rho}
$$

where $\rho$ is the material density (expressed in $\mathrm{kg} / \mathrm{m}^{3}$ ).

\subsection{Profile Update}

The profile update strategy is the set of numerical procedures that allows the calculation of the new wheel profile $w_{n}\left(y_{w}\right)$ (i.e. the profile at the next step), starting from the old wheel profile $w_{o}\left(y_{w}\right)$ (i.e. the profile at the current step) and all the distributions of removed material $\delta_{P^{j k}{ }_{(t)}}(x, y)$. The update strategy, besides evaluating the new profile, is necessary for additional reasons:

1. the necessity to remove the numerical noise that characterizes the distributions $\delta_{P_{w i}^{j k}(t)}(x, y)$ and that, due to non physical alterations of the new profile, can cause problems to the global contact model; 
2. the need to mediate the distributions $\delta_{P_{w i}^{j k}(t)}(x, y)$ in order to obtain a single profile for the wheel (that includes the wear effects of all the wheels of the considered vehicle) as output of the wear model.

The following main steps can be distinguished:

- Longitudinal integration:

$$
\frac{1}{2 \pi w\left(y_{w i}^{j k}\right)} \int_{-a(y)}^{+a(y)} \delta_{P_{w i}^{j k}(t)}(x, y) d x=\delta_{P_{w i}^{j k}(t)}^{t o t}(y)
$$

This operation sums all the wear contributions in the longitudinal direction and spreads the wheel wear along the circumference of radius $w\left(y_{w i}^{j k}\right)$ so to obtain the mean value of removed material for each considered wheel (expressed in $\mathrm{mm}^{3} /(\mathrm{m}$. $\left.m m^{2}\right)$ ).

- Time integration:

$$
\int_{T_{i n}}^{T_{\text {end }}} \delta_{P_{w i}^{j k}(t)}^{\text {tot }}(y) V(t) d t \approx \int_{T_{i n}}^{T_{\text {end }}} \delta_{P_{w i}^{j k}(t)}^{t o t}\left(s_{w}-s_{w i}^{c j k}(t)\right) V(t) d t=\Delta_{P_{w i}^{j k}}\left(s_{w}\right)
$$

the time integration sums all the wear contributes coming from the dynamic simulation to obtain the depth of removed material for the wheel $\Delta_{P_{w i}^{j k}}\left(s_{w}\right)$ expressed in $m m=m m^{3} / \mathrm{mm}^{2}$. In order to have a better accuracy in the calculation of the worn profile, the natural abscissas $s_{w}$ of the curve $w\left(y_{w}\right)$ has been introduced. In particular the following relations locally hold (see Fig. 9):

$$
y \approx s_{w}-s_{w i}^{c j k}(t) \quad ; \quad w\left(y_{w}\right)=w\left(y_{w}\left(s_{w}\right)\right)=\tilde{w}\left(s_{w}\right)
$$

where the natural abscissas of the contact points $s_{w i}^{c j k}$ can be evaluated from their positions $P_{w i}^{j k}$.

- Sum on the contact points:

$$
\sum_{i=1}^{N_{P D C}} \Delta_{P_{w i}^{j k}}\left(s_{w}\right)=\Delta_{j k}\left(s_{w}\right)
$$

where $N_{P D C}$ is the maximum number of contact points of each single wheel. The contact patches are usually less than $N_{P}$ and their number can vary during the simulation; hence, since the summation is extended to $N_{P}$, the contribution of the missing points has been automatically set equal to zero.

- Average on the vehicle wheels and on the dynamical simulations:

$$
\sum_{k=1}^{N_{c}} p_{k} \frac{1}{N_{w}} \sum_{j=1}^{N_{w}} \Delta_{j k}\left(s_{w}\right)=\bar{\Delta}\left(s_{w}\right)
$$

where $N_{w}$ is the number of vehicle wheels while the $p_{k}, 1 \leq k \leq N_{c}, \sum_{k=1}^{N_{C}} p_{k}=1$ are the normalized weights related to the simulations of the statistical analysis, to differentiate the relative impact on the wear of each curve. The average on the number of wheel-rail pairs has to be evaluated in order to obtain as output of the wear model a single average profile for the wheels of the considered vehicle. 
- Scaling:

the aim of the scaling procedure is to amplify the small quantity of material removed during the overall mileage traveled by the vehicle during the $N_{C}$ simulations, that is $k m_{\text {prove }}=l_{\text {track }}$ : in fact, the necessity of acceptable computational time for the multibody simulations leads to adopt small value of the $k m_{\text {prove }}$ length. The chance to take advantage of the scaling lies in the almost linearity of the wear model with respect to the traveled distance.

In this work, a constant discrete step has been chosen to update the wheel profile (see Fig. 10 and equation (27)). This approach requires limited computational load without losing accuracy if compared with different suitable strategies as the adaptive step (due to the almost linearity of the wear model) [6].

If the real chosen mileage $\mathrm{km}_{t o t}$ that the vehicle has to run is divided in discrete steps of length $\mathrm{km}_{\text {step }}\left(\mathrm{km}_{\text {tot }}\right.$ can be chosen depending on the purpose of the simulations, for example equal to the re-profiling intervals) (see Fig. 10), the material removed on the wheel has to be scaled according to the following law:

$$
\bar{\Delta}\left(s_{w}\right) \frac{k m_{\text {step }}}{k m_{\text {prove }}}=\bar{\Delta}^{s c}\left(s_{w}\right)
$$
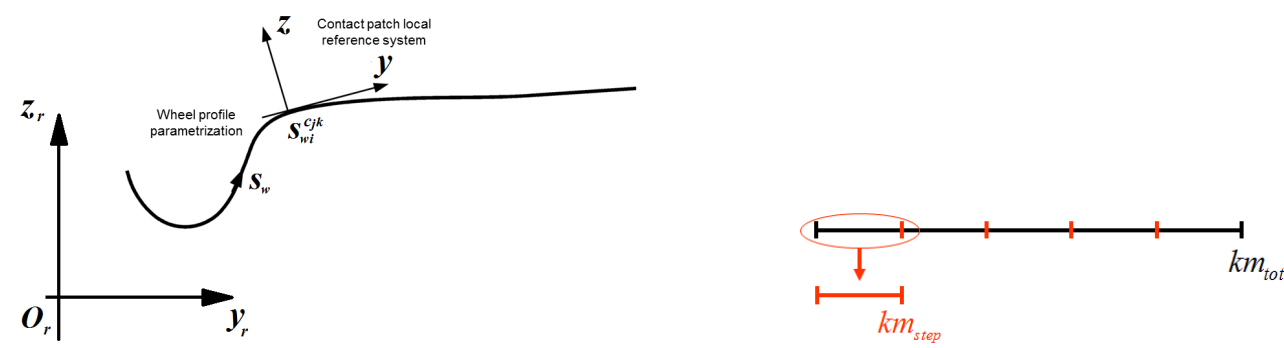

Figure 9: Natural abscissa for wheel and rail profile Figure 10: Discretization of the total mileage

After the scaling, the quantity $\bar{\Delta}^{s c}\left(s_{w}\right)$ is related to a spatial step with a length equal to $\mathrm{km}_{\text {step }}$, instead of $k m_{\text {prove }}$.

The choice of the spatial step must be a good compromise between numerical efficiency and the accuracy required by the wear model. A $\mathrm{km}_{\text {step }}$ too small compared to $k m_{t o t}$ would provide accurate results but excessive calculation times; the contrary happens with $\mathrm{km}_{\text {step }}$ too big if compared to $\mathrm{km}_{t o t}$.

- Smoothing of the removed material:

$$
\Im\left[\bar{\Delta}^{s c}\left(s_{w}\right)\right]=\bar{\Delta}_{s m}^{s c}\left(s_{w}\right)
$$

the smoothing of the removed material function is necessary both to remove the numerical noise and the physically meaningless short spatial wavelengths that affect this quantity (that would be passed to the new profiles $\tilde{w}_{n}\left(s_{w}\right)$ of the wheel causing problems to the global contact model). To this end, a discrete filter (i. e. a moving average filter with window size equal to $1 \% \div 5 \%$ of the total number of points in which the profiles are discretized) has been used. This solution is simple and at the same time the filter does not change the total mass of removed material, as obviously required. 
- Profile update:

$$
\left(\begin{array}{c}
y_{w}\left(s_{w}\right) \\
\tilde{w}_{o}\left(s_{w}\right)
\end{array}\right)-\bar{\Delta}_{s m}^{s c}\left(s_{w}\right) \mathbf{n}_{w}^{r} \stackrel{\text { re-parameterization }}{\longrightarrow}\left(\begin{array}{c}
y_{w}\left(s_{w}\right) \\
\tilde{w}_{n}\left(s_{w}\right)
\end{array}\right)
$$

the last step consists in the update of the old profile $\tilde{w}_{o}(s)=w_{o}(y)$ to obtain the new profile $\tilde{w}_{n}(s)=w_{n}(y)$; since the removal of material occurs in the normal direction to the profile $\left(\mathbf{n}_{\mathrm{w}}^{\mathrm{r}}\right.$ is the outgoing unit vector for the wheel profile), once removed the quantity $\bar{\Delta}_{s m}^{s c}\left(s_{w}\right)$, a re-parameterization of the profile is needed in order to obtain again a curve parameterized by means of the curvilinear abscissa.

\section{Wear Model Validation}

In this section the wear model validation phase will be presented. Initially, the set of $N_{c}$ curvilinear tracks, on which the dynamic simulations of the DMU Aln 501 Minuetto vehicle have been performed, will be introduced; moreover the wear control parameters will be defined (the flange height FH, the flange thickness FT and the flange steepness QR). Then the experimental data measured on the Aosta-Pre Saint Didier track and their processing will be introduced. Finally the results obtained with the wear model will be analyzed and compared with the experimental data.

\subsection{Statistical Analysis of the Aosta-Pre Saint Didier Track}

Starting from the data of the whole Aosta-Pre Saint Didier track (provided by RFI), the statistical analysis has been performed by dividing the line both in radius classes (determined by $R_{\min }$ and $R_{\max }$ ) and in superelevation classes (determined by $h_{\min }$ and $h_{\max }$ ) [7]. More particularly five superelevation subclasses are defined for each radius class. All the $N_{c}$ curved tracks are shown in Tab. 3. Blank rows are present because for certain classes no curves were found. For each of $N_{c}$ class of curves a different constant average worn rail profile provided by RFI has been considered (see Fig. 11); particularly the rail profiles have more pronounced wear as the curve radius becomes narrower because of the contact conditions of the wheel-rail pairs.

The set consists in $N_{c}=18$ distinct elements (17 real curves and the straight line) characterized by the radius value $R_{c}$, the superelevation value $H$, the traveling speed $V$ and the statistical weight $p_{k}$ (with $1 \leq k \leq N_{c}$ ) that represents the frequency with which each curve appears on the considered railway track (Aosta-Pre Saint Didier line). The radii $R_{c}$ are calculated by means of the weighted average on all the curve radii included in that subclass (the weighted factor is the length of the curves in the real track). For each subclass, the value $H$ is the most frequent superelevation value among the values found in that subclass. The traveling speeds $V$ are calculated imposing a threshold value on the uncompensated acceleration $a_{n c}^{l i m}=0.6 \mathrm{~m} / \mathrm{s}^{2}$ :

$$
\frac{\tilde{V}^{2}}{R_{c}}-\frac{H}{s} g=a_{n c}^{l i m}
$$


where $s$ is the railway gauge and $g$ is the gravity acceleration. The estimated speed $\tilde{V}$ has been then compared with the maximum velocity $V_{\max }$ on the line to get the desidered traveling speed $V=\min \left(\tilde{V}, V_{\max }\right)$.

\begin{tabular}{|c|c|c|c|c|c|c|}
\hline $\begin{array}{c}R_{\min } \\
(m)\end{array}$ & $\begin{array}{c}R_{\max } \\
(m)\end{array}$ & $\begin{array}{c}\text { Superelevation } \\
h_{\min }-h_{\max }(\mathrm{mm})\end{array}$ & $\begin{array}{l}R_{c} \\
(m)\end{array}$ & $\begin{array}{c}H \\
(m m)\end{array}$ & $\begin{array}{c}V \\
(k m / h)\end{array}$ & $\begin{array}{l}p_{k} \\
\% \\
\end{array}$ \\
\hline 147.1 & 156.3 & $\begin{array}{c}0 \\
10-40 \\
60-80 \\
90-120 \\
130-160\end{array}$ & $\begin{array}{c}- \\
- \\
- \\
150 \\
-\end{array}$ & $\begin{array}{c}- \\
- \\
- \\
120 \\
-\end{array}$ & $\begin{array}{l}- \\
- \\
- \\
55 \\
-\end{array}$ & $\begin{array}{c}- \\
- \\
- \\
0.77 \\
-\end{array}$ \\
\hline 156.3 & 166.7 & $\begin{array}{c}0 \\
10-40 \\
60-80 \\
90-120 \\
130-160\end{array}$ & $\begin{array}{c}- \\
- \\
- \\
160 \\
165\end{array}$ & $\begin{array}{c}- \\
- \\
- \\
110 \\
140\end{array}$ & $\begin{array}{l}- \\
- \\
- \\
55 \\
55\end{array}$ & $\begin{array}{c}- \\
- \\
- \\
0.48 \\
0.56\end{array}$ \\
\hline 166.7 & $\begin{array}{l}178.6 \\
\end{array}$ & $\begin{array}{c}0 \\
10-40 \\
60-80 \\
90-120 \\
130-160\end{array}$ & $\begin{array}{c}- \\
- \\
- \\
170 \\
175\end{array}$ & $\begin{array}{c}- \\
- \\
- \\
110 \\
130\end{array}$ & $\begin{array}{c}- \\
- \\
- \\
55 \\
55\end{array}$ & $\begin{array}{c}- \\
- \\
- \\
0.82 \\
1.55\end{array}$ \\
\hline 178.6 & 192.3 & $\begin{array}{c}0 \\
10-40 \\
60-80 \\
90-120 \\
130-160\end{array}$ & $\begin{array}{c}- \\
- \\
- \\
190 \\
180\end{array}$ & $\begin{array}{c}- \\
- \\
- \\
100 \\
130\end{array}$ & $\begin{array}{c}- \\
- \\
- \\
55 \\
55\end{array}$ & $\begin{array}{c}- \\
- \\
- \\
8.37 \\
0.45\end{array}$ \\
\hline 192.3 & 208.3 & $\begin{array}{c}0 \\
10-40 \\
60-80 \\
90-120 \\
130-160\end{array}$ & $\begin{array}{c}- \\
- \\
- \\
200 \\
200\end{array}$ & $\begin{array}{c}- \\
- \\
- \\
90 \\
130\end{array}$ & $\begin{array}{c}- \\
- \\
- \\
55 \\
60\end{array}$ & $\begin{array}{c}- \\
- \\
- \\
20.64 \\
4.00\end{array}$ \\
\hline 208.3 & 227.3 & $\begin{array}{c}0 \\
10-40 \\
60-80 \\
90-120 \\
130-160\end{array}$ & $\begin{array}{c}- \\
- \\
220 \\
220 \\
-\end{array}$ & $\begin{array}{c}- \\
- \\
80 \\
100 \\
-\end{array}$ & $\begin{array}{c}- \\
- \\
55 \\
55 \\
-\end{array}$ & $\begin{array}{c}- \\
- \\
0.70 \\
3.76 \\
-\end{array}$ \\
\hline 227.3 & 250.0 & $\begin{array}{c}0 \\
10-40 \\
60-80 \\
90-120 \\
130-160\end{array}$ & $\begin{array}{c}- \\
- \\
240 \\
240 \\
-\end{array}$ & $\begin{array}{c}- \\
- \\
80 \\
110 \\
-\end{array}$ & $\begin{array}{l}- \\
- \\
55 \\
60 \\
-\end{array}$ & $\begin{array}{c}- \\
- \\
7.26 \\
5.28 \\
-\end{array}$ \\
\hline 250.0 & 312.5 & $\begin{array}{c}0 \\
10-40 \\
60-80 \\
90-120 \\
130-160\end{array}$ & $\begin{array}{c}- \\
- \\
270 \\
270 \\
-\end{array}$ & $\begin{array}{c}- \\
- \\
70 \\
90 \\
-\end{array}$ & $\begin{array}{c}- \\
- \\
55 \\
60 \\
-\end{array}$ & $\begin{array}{c}- \\
- \\
3.91 \\
5.29 \\
-\end{array}$ \\
\hline 312.5 & 416.7 & $\begin{array}{c}0 \\
10-40 \\
60-80 \\
90-120 \\
130-160 \\
\end{array}$ & $\begin{array}{c}- \\
- \\
370 \\
345 \\
- \\
\end{array}$ & $\begin{array}{c}- \\
- \\
60 \\
100 \\
- \\
\end{array}$ & $\begin{array}{c}- \\
- \\
55 \\
70 \\
-\end{array}$ & $\begin{array}{c}- \\
- \\
2.26 \\
1.63 \\
- \\
\end{array}$ \\
\hline 416.7 & $\infty$ & 0 & $\infty$ & 0 & 70 & 32.27 \\
\hline
\end{tabular}

Table 3: Data of the curvilinear tracks of the statistical analysis

\subsection{Wear Control Dimensions}

The reference quotas FH, FT and QR are introduced in order to estimate the wheel profile evolution due to the wear without necessarily knowing the whole profile shape . According to these quotas the user will be able both to established when the worn wheel profile will have to be re-profiled and to detect if the wear compromises the dynamical stability of the vehicle [10]. With reference to the Fig. 12 (the quota values are in $\mathrm{mm}$ ) the wear control parameters are calculated as follows: the flange thickness FT is the horizontal distance between the internal vertical face and the point $P 2$; the flange steepness $\mathrm{QR}$ is the horizontal distance between the points $P 1$ and $P 2$, while the flange height FH is the vertical distance between $P 0$ and the flange vertex (all the distances are considered positive).

In regard to their physical meaning, both the flange thickness FT and the flange height FH describe the size of the flange, while the flange height is also a measure 
of the wear on the wheel tread. The QR dimension gives information related to the conicity of the flange.

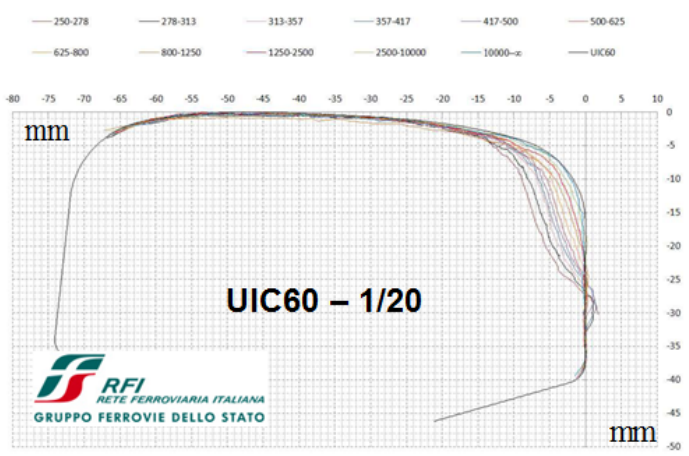

Figure 11: Average worn rail profiles

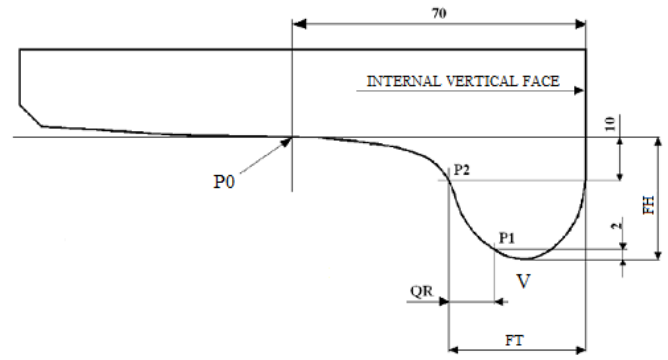

Figure 12: Definition of the wheel wear quotas

\subsection{Experimental Data and Their Processing}

The experimental data provided by Trenitalia and RFI consists in the evolutions of the reference dimensions measured on three different DMUs Aln 501 Minuetto (conventionally named MD061, MD068, MD082) during the service on the AostaPre Saint Didier line.

\begin{tabular}{|c|c|c|c|c|}
\hline Vehicle & $\begin{array}{c}\text { Distance traveled } \\
(\mathrm{km})\end{array}$ & $\begin{array}{c}F H \\
(\mathrm{~mm})\end{array}$ & $\begin{array}{c}F T \\
(\mathrm{~mm})\end{array}$ & $\begin{array}{c}Q R \\
(\mathrm{~mm})\end{array}$ \\
\hline \hline \multirow{4}{*}{ MD061 } & 0 & 28.0 & 32.5 & 10.8 \\
& 1426 & 28.2 & 31.5 & 9.8 \\
& 2001 & 28.1 & 30.8 & 9.1 \\
& 2575 & 28.0 & 30.2 & 8.6 \\
\hline \multirow{4}{*}{ MD068 } & 0 & 28.0 & 32.5 & 10.8 \\
& 1050 & 28.0 & 31.8 & 10.0 \\
& 2253 & 28.0 & 30.2 & 8.5 \\
MD082 & 2576 & 28.0 & 30.0 & 8.4 \\
\hline & 0 & 28.0 & 32.5 & 10.8 \\
& 1800 & 28.0 & 32.3 & 10.6 \\
& 2802 & 28.0 & 31.3 & 9.6 \\
& 3537 & 28.0 & 30.3 & 8.7 \\
\hline
\end{tabular}

Table 4: Experimental data processed

The reference quota values have been measured for all the vehicle wheels (each vehicle has eight wheelsets as specified in section 3.1). However the following data processing has been necessary in order to compare the experimental data with the mean profile extracted from the numerical simulation:

1. initially the arithmetic mean on all the sixteen vehicle wheels, necessary to obtain a single wheel profile and to reduce the measurement errors affecting the experimental data, has been evaluated;

2. then a scaling of the quota values has been carried out to delete the offset on the initial value of the quotas: this procedure imposes that all the wear control parameters start from their nominal values (the standard values for the ORE S 1002 profile 
have been used) in order to remove the initial differences among the vehicles due to measurement errors;

3. the arithmetic mean on the three vehicle MD061, MD068, MD082 has not been carried out, so to maintain a dispersion range for the experimental data.

The experimental data, properly processed, are summarized in Tab. 4. In the numerical simulations, the whole distance traveled available from the experimental data equal to $\mathrm{km}_{\text {tot }} \approx 3500 \mathrm{~km}$, has been divided in $n_{s w}=10$ step, with a resulting $\mathrm{km}_{\text {step }}$ equal to $350 \mathrm{~km}$.

\subsection{Evolution of Wear Control Dimensions}

This section presents the first results of the validation, showing the comparison between the numerically evaluated progresses of the three dimensions (FT, FH and QR) and the treated experimental data. The optimal filter parameters (especially the width of the window) have been picked out to minimize the global error.

The progress of the FT dimension is shown in Fig. 13; as it can be seen, the decrease of the quota is almost linear with the traveled mileage except in the first phases, where the profiles of wheel and rail are not conformal enough.

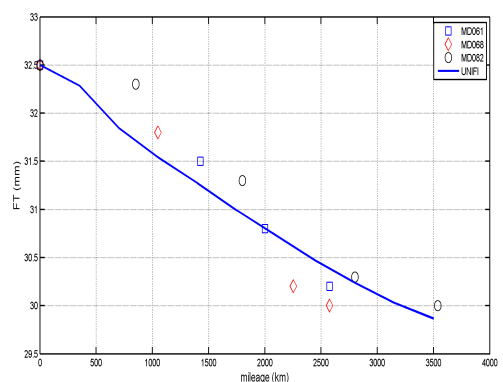

Figure 13: FT progress.

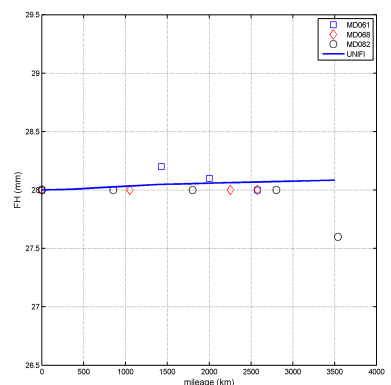

Figure 14: FH progress.

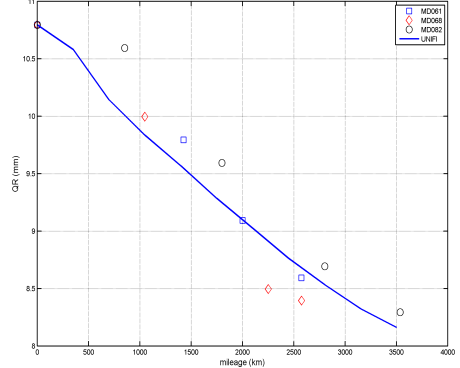

Figure 15: QR progress.

The FH curve progress is presented in Fig. 14 and shows that, due to the presence of many sharp curves in the track and the low traveled mileage, the wear is localized mainly on the flange rather than on the tread; thus the flange height remains nearly constant. The comparison between the real and simulated QR is finally shown in Fig. 15: the dimension decreases almost linearly too, leading to an augmentation of the conicity on the flange. Although the simulated mileage is quite short considering the mean traveled distance between two turning of the wheels in a normal scenery, the variations of the $\mathrm{FH}$ and $\mathrm{QR}$ dimensions are remarkable and evidence difficulties in terms of wear in traveling this railway line with this vehicle.

As a conclusion, the comparisons show that, after the tuning of the filter parameters, the outputs of the wear model are very consistent with the experimental data, both for the flange dimensions (FT, FH) and the QR; therefore the validation of the model can be considered satisfactory. 


\subsection{Evolution of the Wheel Profile}

The numerical evolution of the wheel profile is presented in Fig. 16. Due to the low covered mileage and to the sharpness of the track, the wear is mainly localized on the flange rather than on the tread, where it is quite low and involves a slight reduction of the rolling radius.

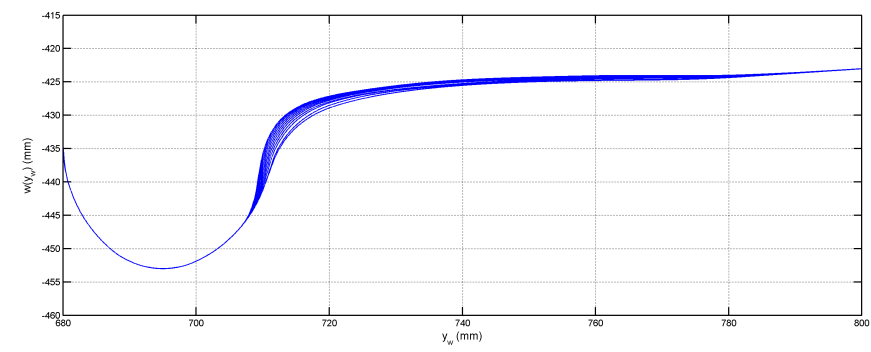

Figure 16: Evolution of the wheel profile.

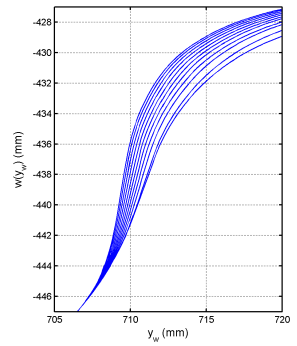

Figure 17: Flange zone.

However, observing the flange zone, the wear rate is higher during the first steps because of the non-conformal contact due to the coupling between the ORE S1002 unworn wheel profile and the UIC60 worn rail with a inclination of 1:20 rad; then it decreases becoming more regular and constant in the last phases, when the contact is more and more conformal. The situation is clarified with the zoom on the flange zone, shown in Fig. 17: the distance between two consecutive profiles drops off as the wear increases.

\section{Conclusions}

In this work the Authors presented a complete model for the wheel wear prediction in railway application, developed thanks to the collaboration with Trenitalia S.p.A and Rete Ferroviaria Italiana (RFI), which provided the necessary technical and experimental data for the model validation. The whole model is made up of two parts which mutually interact. The first one evaluates the vehicle dynamics and comprises both the multibody model of the vehicle and a global wheel-rail contact model (developed by the authors in previous works) for the calculation of the contact points and of the contact forces. The second one is the wear model which, starting from the outputs of the multibody simulations, evaluates the amount of material to be removed for wear. The interaction between the two parts is not a continuos time process but occurs at discrete steps; consequently the evolution of the wheel geometry is described through several intermediate profiles. The developed model reproduces quite good the evolution of all the profile characteristic dimensions describing the wear progress on the wheel.

Future developments may consist in the evaluation of the rail wear evolution and on further experimental data provided by Trenitalia and RFI referred both to railway track with an higher mileage than the Aosta-Pre Saint Didier line and to advanced wear on the wheel (especially on the wheel tread) and on the rail. In this way other analysis will be carried out in order to further validate the whole model. Furthermore a code 
optimization, concerning the vehicle model (in particular the global contact model), the wear model and the whole loop, is scheduled for the future in order to reduce the simulation times.

\section{Acknowledgements}

Authors would like to thank Engg. R. Cheli and G. Grande of Trenitalia S.p.A. for providing and giving the permission to edit the data both to the vehicle DMU Aln 501 Minuetto and to the wheel wear evolution; a special thanks also goes to the Engg. R. Mele and M. Finocchi of Rete Ferroviaria Italiana for the the Aosta-Pre Saint Didier line data.

\section{References}

[1] T.G. Pearce, N.D. Sherratt, "Prediction of wheel profile wear", Wear, 144: 343-351, 1991.

[2] F. Braghin, R. Lewis, R.S. Dwyer-Joyce, S. Bruni, "A mathematical model to predict railway wheel profile evolution due to wear", Wear, 261: 1253-1264, 2006.

[3] E. Meli, S. Falomi, M. Malvezzi, A. Rindi, "Determination of wheel - rail contact points with semianalytic methods", Multibody System Dynamic, 20: 327-358, 2008 .

[4] J.J. Kalker, Three-dimensional Elastic Bodies in Rolling Contact, Kluwer Academic Publishers, Dordrecht, Netherlands, 1990.

[5] J. Auciello, E. Meli, S. Falomi, M. Malvezzi, "Dynamic simulation of railway vehicles: wheel/rail contact analysis", Vehicle System Dynamics, 47: 867-899, 2009.

[6] R. Enblom, M. Berg, "Simulation of railway wheel profile development due to wear influence of disc braking and contact environment", Wear, 258: 10551063, 2005.

[7] P. Toni, "Ottimizzazione dei profili delle ruote su binario con posa 1/20", Technical report, Trenitalia S.p.A., 2010.

[8] A.A. Shabana, M. Tobaa, H. Sugiyama, K.E. Zaazaa, "On the computer formulations of the wheel/rail contact problem", Nonlinear Dynamics, 40: 169-193, 2005 .

[9] S. Iwnicki, "Simulation of wheel - rail contact forces", Fatigue and Fracture of Engineering Materials and Structures, 26: 887-900, 2003.

[10] "Railway applications - In service wheelset operation requirements - In service and off vehicle wheelset maintenance", EN 15313, 2010. 